\title{
IS STI SURVEILLANCE IN ENGLAND MEETING THE REQUIREMENTS OF THE 21ST CENTURY? AN EVALUATION of DAta from the SOUth West Region
}

\author{
C Ihekweazu (chikwe.inekweazu@hpa.org.uk) ${ }^{1},{ }^{2}$, N Maxwell $^{1}$, S Organ $^{1}$, I Oliver $^{1}$ \\ 1. Health Protection Agency South West, Stroud, United Kingdom \\ 2. European Programme for Intervention Epidemiology Training (EPIET)
}

This study evaluates two sexually transmitted infections (STI) surveillance systems' ability to provide relevant, accurate, and timely information to inform prevention and control activities in England, using data from the South West, the largest of the country's nine regions. The systems were evaluated in terms of timeliness of reporting to subsequent levels; frequency of reporting and feedback; completeness of information in the reports; and representativeness of the reports to the resident population. To determine the usefulness of the system for those responsible for taking public health action, semi-structured interviews of a sample of users of surveillance information were conducted. Timeliness of the two main surveillance systems, laboratory reports and returns from genito-urinary medicine clinics were poor. Completeness of the laboratory system was good for date of birth and sex, but poor for geographical markers. Of the 27 respondents that participated in the survey, only eight were satisfied with the level of detail in the surveillance data they received. Most stakeholders felt that the STI data they received was not representative of the population they served and not useful in responding to emerging problems. Faced with increasing incidence of STIs, existing STI surveillance systems in England are unable to provide adequate epidemiological data for the fulfilment of basic uses of public health surveillance at the local level. Surveillance is inadequate in timeliness, geographical coverage, representativeness, does not allow for the identification of risk factors and conceals variations in sex, ethnicity, and sexual behaviour. Disaggregate data with some geographical and riskfactor information would greatly enhance the usefulness of the data. The goal should be of access to real-time data.

\section{Introduction}

Accurate epidemiological information about the occurrence and distribution of sexually transmitted infections (STIs) is imperative for targeted screening, prevention, and control programmes. Recent syphilis outbreaks in the South West region of England [1,2], as well as increasing demands by frontline health staff and Primary Care Trusts for detailed information have highlighted shortcomings in the surveillance data presently available to public health professionals on STIs.

The rates of STIs in England have been on the increase over the past few years [3] and there is a need to understand the factors driving this to inform the public health response. Genital chlamydial infection is the most commonly diagnosed STI in genitourinary medicine (GUM) clinics and genital herpes the most common cause of ulcerative STIs in England. Since 1995, diagnoses of gonorrhoea in England have been rising again after a declining considerable since 1985 [4].
Improving sexual health has been a priority of the UK government and the need for action was reinstated in its recently published white paper, "Choosing Health: Making Health choices easier" [5].

Surveillance of STIs should provide relevant, accurate, and timely information to inform prevention and control activities. It should also be able to provide population estimates for prevalence and incidence, trends, identify risk factors, and present information in a timely and assessable manner [6]. This study evaluates STI surveillance in the South West region of England to ascertain if it is meeting these objectives.

\section{Methods}

\section{STI surveillance in England}

There are two main sources of STI data in England: genitourinary medicine (GUM) clinics and microbiology laboratories.

Data collected from all GUM clinics, off-shoots of veneral disease clinics, led to the UK's first systematic STI surveillance system [7]. These GUM clinics are open access and offer free, confidential sexual health services. Data statutorily submitted quarterly from these clinics are known as the KC60 returns. KC stands for Korner Code, an abbreviation given to statutory returns from the NHS to the Department of Health; for example, KC62 is breast cancer, KC64 dental activity and KC51 immunisation uptake. This system was initially developed as a tool for workforce planning and monitoring clinic activity. It was set up in 1917 as a system for measuring workload at GUM clinics in the country. This system is supplemented by a second surveillance system called CoSurv. This involves the voluntary laboratory reporting of STIs from the laboratories through regional office to the national centre.

The aim of this study was to evaluate the performance of these two surveillance systems for STIs in England between 1997 and 2004 using data from the South West region in order to determine the systems' ability to provide high quality data in a timely and efficient manner. Our evaluation focused on the needs of local and regional users of STI surveillance information in this region. The flow of data through the two surveillance systems is described below.

As a result of the perceived inadequacies of STI surveillance, the Enhanced Surveillance for Infectious Syphilis Programme, a new national surveillance system, was established to better determine and describe the geographic, demographic and risk factor distribution of infectious syphilis. This initially collected data from all GUM clinics in London with the aim of extending 
it to all GUM clinics in England by 2002. However this has not been undertaken due to lack of resources centrally. In response to Syphilis outbreaks in the South West, a similar system was initiated in the region [8]. As this was implemented just before this evaluation, syphilis was excluded from the evaluation due to the likelihood of surveillance artefacts.

\section{The KC60 system}

All 206 clinics in England have a statutory obligation to complete an aggregate statistical return on their attendance called the KC60 returns. These data on the total numbers STI episodes seen (individuals may be included more than once) are collected by each GUM clinic. There are 19 GUM clinics spread across the South West. Data are aggregated by age-group, sex and number of cases in men that were homosexually acquired. Each diagnosis is assigned a KC60 code. The data are sent quarterly from the clinics directly to the national Centre for Infections of the Health Protection Agency (HPA) for analysis. The lowest level of geographical data available is the clinic level, for which the catchment populations are unknown. Data are disseminated via an annual STI report, and through ad hoc requests. A subset of data is also sent to the regions annually for analysis and further dissemination to local users.

\section{The laboratory-based system (CoSurv)}

Laboratory reports are received from all the 16 laboratories in the South West region. These laboratories receive samples from primary and secondary care providers that cover the entire population accessing the National Health Service in the region.

F I G U R E 1

Flowchart of KC60 surveillance system for the South West of England, 2005

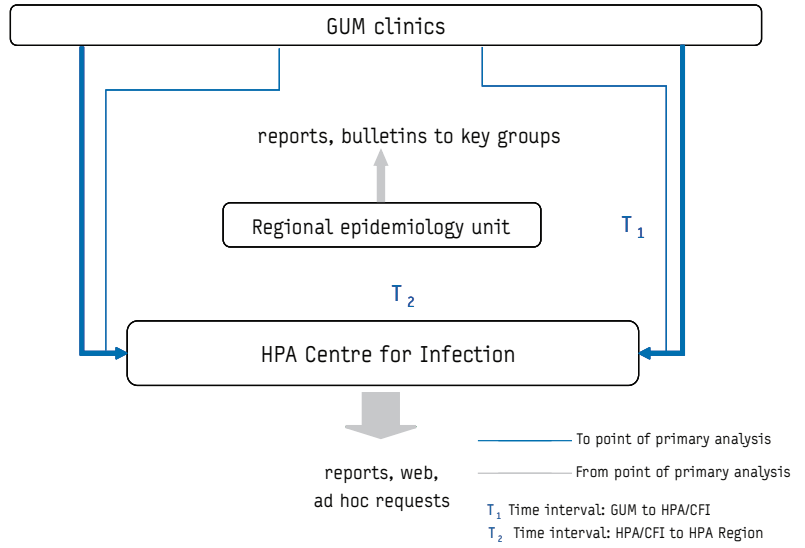

Reporting is electronic and can be done at anytime. These reports are then sent to the HPA Centre for Infection. Staff at the national centre scan the data weekly using specifically designed algorithms to detect increases in the number of reports above what would be expected based on data from the previous five years and produce the "Exceedance reports" [9]. These exceedance reports sent down to the regions weekly alerts staff to temporal and geographic clusters and triggers further investigation.

The US Centers for Disease Control and Prevention's Updated Guidelines for Evaluating Public Health Surveillance Systems [10] was used as the main tool for the evaluation methodology. This tool guides the measurement of the attributes of surveillance systems such as well as the degree to which
F I G U R E 2

Flowchart of laboratory based surveillance system for the South West of England, 2005

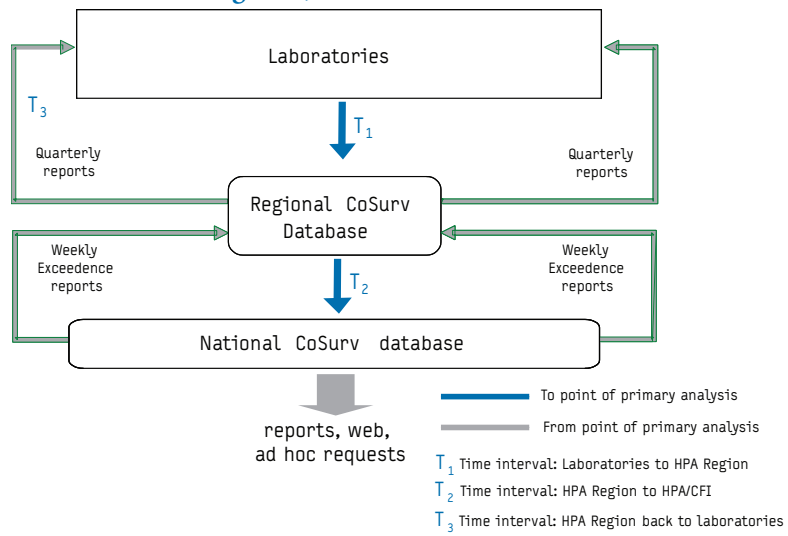

the data produced is used to stimulate public health action. This evaluation was commissioned South West Sexual Health Task Group. Our evaluation was limited to those attributes that were considered of highest priority to local needs and priorities, the peculiarities of STIs, and the availability of data; these included timeliness, completeness, representativeness, and usefulness.

The timeliness and completeness of data transfer from the collection to dissemination stages were evaluated to ascertain that surveillance outputs are both adequate and timely enough to trigger appropriate public health action. Timeliness and completeness for both systems were evaluated for the period 1997 to 2004 .

Timeliness of reports to CoSurv was evaluated by calculating the mean number of days from the date of specimen collection from patients to the date of availability of results in the regional database, the mean number of days until results were available in national database and the number of weeks it took for a report on the data to be available for public health action. For the KC60 system, the mean number of weeks from the end of each quarter until the entry of data into the national database from GUM clinics (T1 in Figure 1) and the time it takes for analysis of the data from GUM clinics to be done at the national level and reported to the regions (T2 in Figure 1) was calculated. Completeness of reports to CoSurv was evaluated by calculating the proportions of reports that had complete information on age, sex, postcode of residence and the GP postcode (the postcode of the general practitioner where the patient is registered).

The representativeness of the KC60 system was evaluated by comparing the data from a similar geographical area to the Avon Surveillance System for Sexually Transmitted Infections (ASSIST) [11, 12]. ASSIST was established as a research project to explore the feasibility of collecting disaggregate data from all STI service providers in the Avon area, which makes up a fifth of the population of the South West region of England. This was done by integrating genitourinary clinic and laboratory data. Postcode information for geographical mapping and small area analysis was obtained by matching pseudo-anonymised data with GP registration databases. 
T A B L E 1

Numbers of each stakeholder group interviewed

\begin{tabular}{|c|c|c|}
\hline Group & $\begin{array}{c}\text { Number of members of } \\
\text { group in SW region }\end{array}$ & Number surveyed \\
\hline GUM Physicians & 19 & 5 \\
\hline $\begin{array}{c}\text { Consultants in } \\
\text { Communicable Disease } \\
\text { Control (CCDCs) }\end{array}$ & 12 & 6 \\
\hline $\begin{array}{c}\text { Consultants in Public } \\
\text { Health }\end{array}$ & 30 & 5 \\
\hline $\begin{array}{c}\text { Consultant } \\
\text { Microbiologists }\end{array}$ & 16 & 3 \\
\hline $\begin{array}{c}\text { Members of the regional } \\
\text { epidemiology team }\end{array}$ & 8 & 24 \\
\hline Total & 85 & 2 \\
\hline
\end{tabular}

The usefulness and perceptions of the surveillance systems' attributes were evaluated through semi-structured interviews of a sample of suppliers and users of STI surveillance data. A proportionate stratified random sampling method was used for selecting the respondents for the structured telephone interviews used for this study [11]. We randomly selected approximately $25 \%$ of all the members of each group of stakeholders (Table 1) to be interviewed. The respondents were chosen from their professional lists in the South West region. A breakdown of the members in each individual group interviewed is given below. For voluntary organisations working on STIs in the region, we randomly selected three of the nine organisations known to us and spoke to the most senior member of staff on the day we telephoned.

The answers to the structured questions were entered into an Epidata database for descriptive frequency analysis and proportions calculated. The answers to the open-ended questions were reported verbatim and subsequently reviewed and organised into specific themes. These were then reviewed in relevance to the related attribute.

\section{Results}

Timeliness

The KC60 system

The mean number of weeks from the end of each quarter until the entry of data into the national database from GUM clinics (T1 in Figure 1) decreased from a mean of 24 weeks in 2003 to 18 weeks in 2004. Considerable variation exists in the timeliness of individual clinics sending in their quarterly returns and as a result, these are collated, and only entered into the national database when the deadline for all clinics to report has elapsed. An analysis of a subset of the data from GUM clinics is done for the regional and clinic level annually at the national level and a report is sent to regions. On average (between 1998 and 2003), this report reached the region 29 weeks after the end of the year. Accounting for the reporting delay for data to get to the national centre (mean $=21$ weeks based on 2003/2004 data (T1)), it took an additional eight weeks to report this data to the regional level (T2). Therefore it takes between seven months and 19 months to receive data relating to cases that were reported in the KC60 system depending on which end of the year it was reported.
F I G U R E 3

Median number of days between specimen date entry into regional database

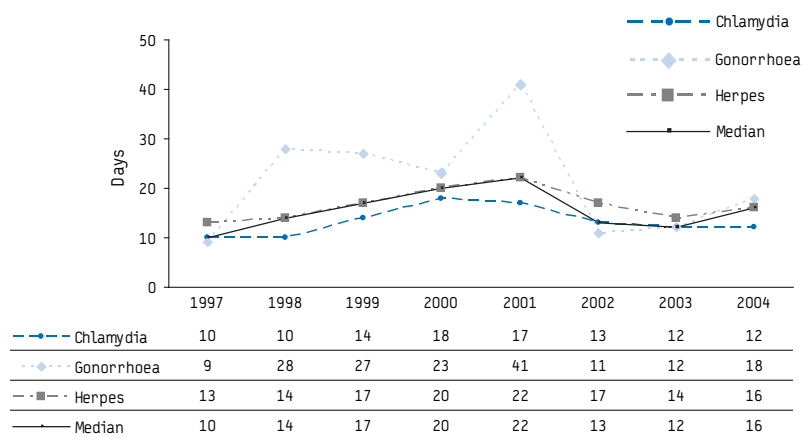

\section{The laboratory system}

Between 1997 and 2004, there were 90,087 reports of genital herpes, gonococcal and genital chlamydia infections in the regional database. The point T1 in Figure 2 illustrates the period between the date of collection of the organism and its entry into the regional database. Over the eight years, the median number of days it took for data to enter the regional database was 15 and an additional two days to enter the national database (T2 in Figure 2). In the South West of England, a report based on the data is produced and sent out to stakeholders at the end of each quarter. The time (T3 in Figure 2) between the end of the quarter and the date of data dissemination through a report is on average eight weeks. In summary, it takes between eight and 21 weeks for laboratory to become available for dissemination.

\section{Perception of timeliness of both systems}

Of the 27 stakeholders that responded to the survey, 20 stated that the data they receive is not timely enough to respond to emerging problems. Four respondents were satisfied with the frequency of receiving the KC60 data (sent out annually) while 11 were satisfied with the frequency of the laboratory data (sent out quarterly) received. Most respondents would prefer to receive data quarterly.

\section{Completeness}

\section{The KC60 system}

Since data received through this system is in aggregate form, completeness could only be analysed in terms of the number of GUM clinics sending their data to the national level. In this respect, given the generous intervals allowed to GUM clinics to report, completeness is close to $100 \%$. Considering the last two years of the evaluation; 2003 and 2004, 95\% of all quarterly returns from the 19 GUM clinics were entered into the national database for each quarter before the deadline for that quarter. Evaluating completeness in terms of individual STIs is only possible by auditing data at the clinic level, which was beyond the scope of this study.

\section{The laboratory system}

There were 92,007 reports of genital herpes, gonorrhoea and genital chlamydia cases between 1997 and 2004 from all 16 laboratories in the region (10 for genital herpes). In 2004, 12,282 genital chlamydia cases were reported via the CoSurv system, 
F I G U R E 4

New episodes of genital herpes, gonorrhoea and genital chlamydia in the South West region of England, 1997 to 2004 reported via CoSurv

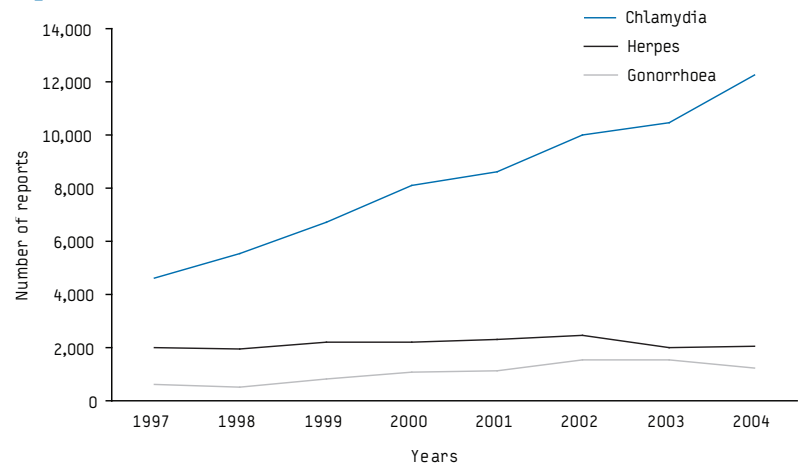

an increase of $265 \%$ on the 4,624 cases in 1997 . Reports of gonorrhoea also increased steadily since 1997 . A total of 1,218 cases were reported in 2004 a 203\% increase compared to 1997 . The rise in reported genital herpes cases was less marked, with 2,000 cases in 1997 and 2,071 in 2004

Completeness was evaluated for the information contained in each report. The completeness of reports for gonorrhoea and genital chlamydia infections was assessed for all 16 laboratories and for 10 of the 16 laboratories reporting genital herpes consistently over the past eight years (not all laboratories perform herpes diagnosis). Completeness for date of birth and age was above $90 \%$, but below $30 \%$ for the two geographical variables, postcode and GP postcode (see Figure 5), however there has been some improvement in the completeness of these variables in the past five years.

\section{Representativeness}

To assess the representativeness of the KC60 system, we compared surveillance data from the KC60 returns to ASSIST for an equivalent population and time period [12]. ASSIST collected disaggregate data from all STI service providers in the Avon area. Results from this project showed that just $31 \%$ of genital chlamydia and $64 \%$ of gonorrhoea diagnoses were captured by the KC60

F I G U R E 5

Completeness of genital herpes, gonorrhoea and genital chlanydia reports in regional database, for the South West of England, 1997 to 2004

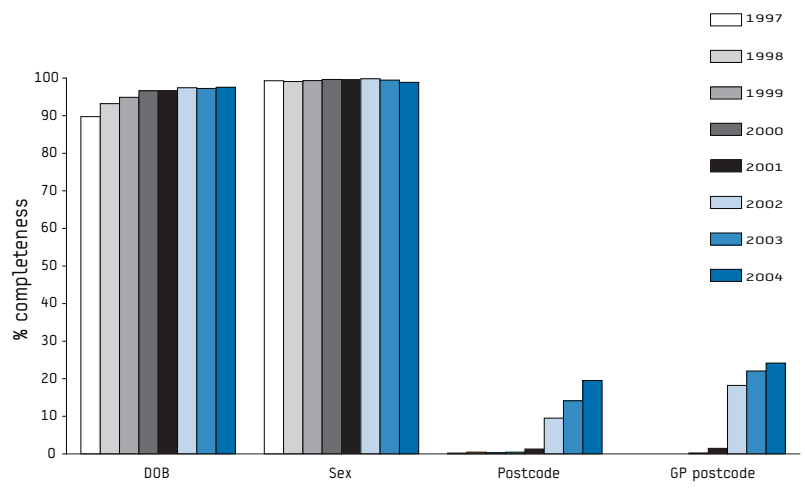

returns from GUM clinics alone when compared to data from all sources in the areas served by ASSIST in 2002 [13].

\section{Perception of representativeness}

Most respondents (24/27 for KC60 and 22/27 for CoSurv) felt that the STI data they received was not representative of the population they served. The reasons mentioned for this perception includes that the KC60 data only represents people presenting at GUM clinics with no data from primary care settings. Also mentioned was the perception of significant patient mobility and preference of patients for open-access clinics.

\section{Usefulness}

Of the 27 respondents that participated in the survey, 24 received any STI surveillance data and only eight received outputs from both systems. While public health physicians at the local and regional level have good access to outputs of both sources of STI data, GUM physicians only received KC60 outputs while microbiologists only received laboratory outputs, i.e. each group only receiving the data that they are directly involved with. Voluntary organisations involved in activities promoting sexual health were not receiving any outputs from either of the two STI surveillance systems.

Only 13 of the 27 respondents found the geographical level of the data they receive at as adequate. The geographical level at which data was most desired by respondents was the 'Primary Care Trust' level: 11 of the 13. This is the level at which health services are commissioned for the population. The level of detail in the STI data received was satisfactory according to eight respondents. Additional information most desired was additional geographical and risk factor information. Twenty of the 27 said that they discussed STI data regularly at a local forum, often a sexual health strategy group. The use to which the STI data is put is listed below in order of the frequency with which the 27 respondents mentioned it:

\section{Discussion}

The main objectives of disease surveillance systems are the efficient collection, collation and analysis of high-quality data, regular provision of feedback to the participants who provided the data (as well as to any relevant stakeholders and decision-makers), in order to enable the implementation of appropriate public health action. In addition to the above, a surveillance system should be

TA B L E 2

Uses for STI data according to 27 respondents in the South West of England

\begin{tabular}{|l|c|}
\hline Use & Number of times mentioned* \\
\hline Monitor trends & $8(30 \%)$ \\
\hline Plan service delivery & $7(26 \%)$ \\
\hline Dissemination & $4(15 \%)$ \\
\hline Plan prevention & $3(11 \%)$ \\
\hline Training & $3(11 \%)$ \\
\hline Use for strategy meetings & $3(11 \%)$ \\
\hline Compare with other areas & $3(11 \%)$ \\
\hline Scan for news stories & $1(4 \%)$ \\
\hline Trigger research question & $1(4 \%)$ \\
\hline
\end{tabular}

*Multiple choices were possible. 
able to detect changes in incidence and prevalence in groups of people most at risk of infection, so that targeted prevention and intervention strategies can be developed [7].

For both STI surveillance systems evaluated, timeliness was a major problem. The KC60 data was received at the regional level from the national level annually (midway into the year after it is collected) from where it is further disseminated to users by the regional epidemiology team. The majority of the users we surveyed felt that the data generated was not useful in reacting to emerging problems as a result of the lateness of the reports. This lateness has significantly undermined the confidence of users, as illustrated by the survey.

While completeness of reports was very good for age and sex in the laboratory system, it was extremely poor for the geographical markers. This limits its value for analysis to be done at relevant geographical levels. Primary Care Trusts, which have the responsibility of commissioning prevention services for their populations, need data at this geographical level for the justification and evaluation of programmes/projects.

The dominant view among stakeholders surveyed was that, other than in broad population terms, the data available was poorly representative. The absence of data from primary care settings, and patients' preference for open-access clinics is believed to skew the data. With the increasing burden of genital chlamydia and the increasing access to sexual health services in settings such as pharmacies, the reliance on GUM clinics for STI data might be inadequate for measuring the burden of STIs in the population.

Many respondents wanted information on risk factors, demography, ethnicity and occupation. Studies have shown that the burden of STIs disproportionately affects certain subpopulations [14, 15] and identifying groups at greatest risk will enable interventions to be targeted. The current, aggregate system of STI data collection in England is unable to fully explain these differences and it will be difficult to show progress in all the new initiatives to improve sexual health.

The strength of the KC60 system is in its stability (not explicitly analysed in this paper), its long-standing existence (since 1917) and therefore its reliable trend data. The collection of minimum risk factor information was desired by most respondents as the data at present conceals variations in respect to sex, ethnicity, and sexual preference.

Faced with an increasing incidence of STIs, surveillance in England is not fulfilling one of the fundamental goals of infectious disease surveillance; to provide information for action. Some of these issues might be addressed with the introduction of the minimum data set for STIs when the national programme for information technology is complete [6]. But in the meantime there is a need to provide timely and useful information for planning, prevention and intervention evaluation.

In attempting to address similar problems, some other regions in England have adapted their surveillance by setting up parallel systems to collect disaggregate data from GUM clinics [16]. Elsewhere, Scotland has recently set up a web-based system STISS (STI Surveillance Scotland) providing real-time secure data collection and validation with scalability functions to any number of sites [17]. All clinics were given NHS-net-enabled computers (secure access to the NHS network); and diagnostic codes were revised to introduce service codes, yielding denominator data. This has led to significant improvements in timeliness and completeness. Other advantages include: real-time secure data collection; realtime validation, enhancing data completeness and accuracy; context-sensitive help; flexible revisions to codes; scalability to any number of locations with minimum site visits. [18].

Several other European countries have recently reported improvement since resorting to web-based electronic reporting for all infectious diseases [19, 20]. Significant improvements in timeliness and completeness of surveillance data were reported following the change to an Internet-based reporting system in the Netherlands[18], while an increased detection of outbreaks was reported in Germany[19].

The arrangement in the England which has separate clinics exclusively for the management of STI, would be a perfect example of the scalability of one system across different treatment settings. A web-based surveillance system would ultimately be needed for the capture of data from different types of service providers. Varying access limitation would overcome issues of confidentiality, allowing the collection of disaggregate data. A web interface would also allow for appropriate data extraction and the ability to interactively analyse up-to-date epidemiological data. The goal should be access to real-time data.

The scope of our analysis is limited the use of data from only one of England's nine regions. However, we feel that confident that the findings represent the situation across the country and our data provides a unique perspective of the relevance of a national surveillance system for public health action at the local level.

\section{Acknowledgements}

Many thanks to all GUM Physicians, Consultants in Communicable Disease Control, Consultants in Public Health, Microbiologists and members of the South West Regional Epidemiology Unit that responded to our questions and also thanks to Valerie Delpech and Catherine Lowndes for their kind review of our protocol.

\section{References}

1. CDSC, An outbreak of infectious syphilis in Bristol. Commun Dis Rep CDR Wkly, 1997. 7(33): p. 291.

2. Health Protection Agency, Outbreak of Infectious Syphilis in Gloucestershire. CDR Weekly, 2004. 15(13).

3. Health Protection Agency HIV \& STI division, The Surveillance of HIV/AIDS, other Sexually Transmitted Infections and Associated Behaviour: A description of current methodology. 2003.

4. Health Protection Agency. 2005 Epidemiological Data - Gonorrhoea. Available from: http://www.hpa.org.uk/infections/topics_az/hiv_and_sti/sti-gonorrhoea/ epidemiology.htm

5. Department of Health, Choosing Health: Making healthy choices easier. Available from: http://www.dh.gov.uk/assetRoot/04/09/47/51/04094751.pdf

6. Simms, I et al., Surveillance of sexually transmitted infections in primary care. Sex Transm Infect, 2003; 79: 14-179.

7. PHLS/ DHSS\&PS/ ISD(D) 5 Collaborative Group, Sexually Transmitted infections in the UK: New episodes seen at Genitourinary Medicine Clinics 1991 - 2001. 2001.

8. Oliver I, Christensen $\mathrm{H}$. Two epidemiologically distinct syphilis outbreaks in south west England detected in 2004 with few apparent links to outbreaks in urban areas. Euro Surveill 2005;10(1):E050127.4. Available from: http://www.eurosurveillance.org/ ew/2005/050127.asp\#4

9. Farrington, C.P. and A.D. Beale, Computer-aided detection of temporal clusters of organisms reported to the Communicable Disease Surveillance Centre. Commun Dis Rep CDR Rev, 1993. 3(6): p. R78-82. 
10. Center for Disease Control and Prevention, Updated Guidelines for Evaluating Public Health Surveillance Systems. MMWR, 2001. 50(13): p. 1-35.

11. Patton, M.Q., Qualitative evaluation and research methods (2nd ed.). 1990, Newbury Park CA: Sage Publications.

12. Slater, W. and N. Low, Surveillance of sexually transmitted infections in primary care. Sex Transm Infect, 2004. 80(2): p. 152

13. Slater W, Low N for the ASSIST Project Group. Avon Surveillance System for Sexually Transmitted Infections. Eastbourne: Faculty of Public Health Medicine Annual Scientific Meeting, June, 2003:24-6.

14. Fenton, K.A., et al., Ethnic variations in sexual behaviour in Great Britain and risk of sexually transmitted infections: a probability survey. Lancet, 2005. 365(9466): p. 124655.

15. Rice, B.D., et al., The changing epidemiology of prevalent diagnosed HIV infections in England, Wales, and Northern Ireland, 1997 to 2003. Sex Transm Infect, 2005. 81(3): p. 223-9.

16. Blair, I., et al., Surveillance of sexually transmitted infections in the West Midlands using anonymised individual patient datasets from genitourinary medicine clinics. Commun Dis Public Health, 2004. 7(2): p. 112-9.
17. NHS Scotland STI Surveillance System Support. http://www.show.scot.nhs.uk/support/ sti/index.htm (Accessed 02/08/05).

18. Winter $A J$ and Thompson $C$ on behalf of the STISS steering group. STISS: developing a national web-based STI coding system in Scotlan. Dublin: 11th Annual Conference of the British HIV Association ; (abstract P125)

19. M Ward, P Brandsema, E van Straten, A Bosman. Electronic reporting improves timeliness and completeness of infectious disease notification, The Netherlands, 2003. Euro Surveill 2005;10(1):27-30. Available from: http://www. eurosurveillance.org/em/v10n01/1001-224.asp

20. Faensen D, Claus H, Benzler J, Ammon A, Pfoch T, Breuer T, and al. SurvNet@ RKI - a multistate electronic reporting system for communicable diseases. Euro Surveill 2006;11(4):100-3. Available from: http://www.eurosurveillance. org/em/v11n04/1104-224.asp

Citation style for this article: Inekweazu C, Maxwell N, Organ S, Oliver I, . Is STI surveillance in England meeting the requirements of the 21 st century? An evaluation of data from the South West Region. Euro Surveill 2007;12(5)[Epub ahead of print]. Available online: http://www.eurosurveillance.org/em/v12n05/1205-225. asp 